\title{
Multiple sources of positive- and negative-priming effects: An event-related potential study
}

\author{
HENNING GIBBONS, THOMAS H. RAMMSAYER, and JUTTA STAHL \\ University of Göttingen, Göttingen, Germany
}

\begin{abstract}
Event-related potential correlates of positive priming (PP) and negative priming (NP) were investigated in order to further elucidate the cognitive mechanisms involved. Thirty-six participants performed both an identity- and a location-based priming task. Repeating the target stimulus/location from the immediately preceding display produced behavioral PP. With localization, but not with identification, behavioral NP was observed when the target stimulus/location matched the preceding distractor stimulus/location. Smaller P300 amplitude accompanied identity-based PP, suggesting persisting target-specific activation. The lateralized readiness potential, an index of correct/incorrect response activation, indicated persisting central motor activation as another source of PP. Both location-based PP and NP were accompanied by reduced P1/N1 and P300 amplitudes, pointing to the involvement of inhibition of return in location-based priming. The results support the view that multiple brain processes underlie behavioral priming.
\end{abstract}

Responding to a repeated target stimulus or target location (attended repetition, AR) is often facilitated, as is indicated, for instance, by shorter response times (RTs), as compared with a control condition in which there is no repeated target presentation. This effect is usually referred to as repetition priming or positive priming ( $\mathrm{PP}$; see Tenpenny, 1995, for a review). On the other hand, responding to a target stimulus that has been ignored immediately before or appears in a location that has been ignored immediately before (ignored repetition, IR) is often delayed or more error prone, as compared with a control condition. The latter finding has received much attention and is known as the negative-priming effect (NP; Tipper, 1985; for reviews, see Fox, 1995a, and May, Kane, \& Hasher, 1995).

A typical experimental paradigm, which can be used to obtain PP and NP effects, is the Eriksen flanker task (Eriksen \& Eriksen, 1974). With this task, participants are presented with letter triplets containing one central letter and two identical flanking letters that are different from the target letter. Their task is to press, as quickly as possible, a response button assigned to the central letter and to ignore the flankers. A trial always consists of two subsequent displays of letter triplets, referred to as the prime and the probe. In AR trials, in which the target stimulus (i.e., the central letter) is the same in both the prime and the probe, PP should be observed in terms of a decreased probe RT and/or fewer errors, as compared

The authors thank Bruce Milliken and an anonymous reviewer for their helpful comments on an earlier draft of this article. Correspondence concerning this article should be addressed to H. Gibbons, Georg-EliasMüller-Institute for Psychology, University of Göttingen, Gosslerstr. 14, D-37073 Göttingen, Germany (e-mail: hgibbon@uni-goettingen.de). with control trials, in which there is no relation between the letters in the prime and the probe. Similarly, IR effects can be tested using a condition in which the flanker letter from the prime becomes the target in the probe. NP, if observed, refers to an increased probe RT and/or more errors in the IR condition than in the control condition. PP and NP have been demonstrated with several variations of the flanker task (Driver \& Tipper, 1989; Fox, 1995b; Stadler \& Hogan, 1996).

PP and NP can also be shown with localization tasks. In the typical location suppression task (Tipper, Brehaut, \& Driver, 1990), the primes and probes consist of four marked locations, one of which is occupied by a letter $\mathrm{X}$, and one by a letter $\mathrm{O}$. Participants have to respond as quickly as possible by pressing the key that corresponds to the location of the target letter $\mathrm{O}$ and to ignore the distractor letter X. In AR trials, the probe contains a target $\mathrm{O}$ that is repeated at the location at which it appeared in the prime, whereas in IR trials, the probe target appears at the location at which the prime distractor was presented. In control trials, the target and distractor stimuli of the probe are presented in two locations that were not occupied in the preceding prime. Whereas robust NP effects could be established for IR trials, in numerous studies no PP effect has been observed for AR trials (Connelly \& Hasher, 1993; Milliken, Tipper, Houghton, \& Lupiáñez, 2000; Park \& Kanwisher, 1994; Shapiro \& Loughlin, 1993; Tipper et al., 1990).

There are three major theories of priming effects: the activation/inhibition view (Houghton \& Tipper, 1994; Morton, 1969, 1979; Tipper \& Cranston, 1985), the episodic retrieval view (Neill \& Valdes, 1992; Neill, Valdes, Terry, \& Gorfein, 1992), and the feature match/mismatch theory (Milliken, Tipper, \& Weaver, 1994; Park \& Kanwisher, 1994). All three of these theoretical frameworks 
have been derived from the literature on NP. It should be noted, however, that each of these frameworks for interpreting NP probably stems from earlier proposals about priming in general (e.g., Logan, 1988, in the case of the episodic retrieval view). The activation/inhibition view (e.g., Houghton \& Tipper, 1994; Tipper \& Cranston, 1985) assumes that a process occurring in the prime - namely, selection of a target against a distractor - is responsible for priming effects. Since, in the prime, a target and a distractor are presented, processing of the target is facilitated, while concomitantly, processing of the distractor is inhibited, so that the correct response may be selected efficiently. In an immediately following probe, processing of the target benefits from persisting activation, in cases in which there is a match with the prime target. On the other hand, processing of a probe target is delayed in cases in which there is a match with the prime distractor, reflecting persisting inhibition of the prime distractor.

According to the episodic retrieval view (Neill et al., 1992), priming effects are caused mainly by processes occurring during probe presentation. Stimuli (or locations) repeating from the prime provide cues for retrieving information about these stimuli (or locations) and their associations with responses. Consequently, on AR trials, processing of the probe target stimulus benefits from retrieval of correct stimulus-response information from the prime. On the other hand, on IR trials, processing of the probe target stimulus is delayed, since there is a conflict between the information retrieved from the prime ("Do not respond to this stimulus") and the current stimulus evaluation, in which that stimulus is considered as a target ("Do respond to this stimulus").

Feature match/mismatch theory (Park \& Kanwisher, 1994 ) is limited to explaining priming effects in localization tasks (but see MacDonald \& Joordens, 2000). It is claimed that PP occurs with AR trials because the same stimuli are repeated in the same locations in both the prime and the probe, irrespective of their status as target or distractor. NP observed with IR trials is explained by the fact that the same stimuli appear in different locations in the prime and the probe, irrespective of their status as distractor or target, thereby causing feature mismatch. Thus, feature match facilitates feature analysis and produces PP, whereas feature mismatch delays feature analysis and produces NP.

Although there are a large number of studies on priming and event-related potentials (ERPs), no conclusion can be drawn from these studies with regard to the validity of the three major theories of priming above. First, many ERP studies have investigated priming effects from nonidenti$\mathrm{cal}$ prime and probe stimuli that varied in semantic relatedness (semantic priming; see, e.g., Kiefer, 2002; Rossell, Price, \& Nobre, 2003). Second, even if identical prime and probe stimuli have been used in ERP studies on repetition priming, both the methods and the goals of these studies were quite different from those in the present study. Typically, many different stimuli (words) were presented, with one study opportunity per stimulus, and the interest was in retrieval from episodic memory - that is, whether or not participants correctly recognized a word on its second presentation as having occurred earlier (see Rugg, 1995, for a review). This type of task strongly differs from the tasks usually employed in the literature on identity and location priming, including the present study, where speeded responses have to be performed in response to the identity or location of a small number of simple target stimuli. Third, ERP priming tasks usually do not involve selection from different objects, which is generally considered the only situation in which all three major theories of priming may hold (Fox, 1995a). Location-based ERP priming tasks, such as cuing (Eimer, 1995), also do not involve selection processes and, in addition, usually lack a control condition. Hence, the priming effects observed in previous ERP studies cannot be directly related to the three major theoretical accounts of identity- and location-based priming effects. Therefore, the aim of the present study was to investigate ERP correlates of PP and NP processes obtained with tasks relating more strongly to the experimental priming literature. By the use of ERP methods, we attempted to obtain electrophysiological information, in addition to the mere behavioral data, which may help to further evaluate the three different priming theories.

Feature match/mismatch theory assumes that (locationbased) priming results from faster/slower perceptual processes if the same locations are occupied by the same/ different stimuli in both the prime and the probe displays. Consequently, early ERP components reflecting early visual analysis, such as posterior P1 and N1 components, should be sensitive to experimental conditions. This line of reasoning implies that the processing delay that occurs in visual feature analysis will be transmitted to the behavioral response without affecting stimulus processing at later stages. Thus, the latency, rather than the amplitude, of later ERP components (e.g., the P300 component) should vary with experimental conditions. It is important to note that feature match/mismatch theory predicts the opposite early ERP differences from the control for the AR and IR conditions. For example, if P1 peak latency were shorter for AR than for control trials, it should be longer for IR than for control trials.

On the other hand, if behavioral priming is due exclusively to activation/inhibition processes, separate predictions must be set up for localization and identification tasks. When activation/inhibition refers to a location, attention should be more/less easily directed to that location, and priming effects on ERPs can be expected to be similar to results from earlier visuospatial-cuing and selective attention ERP studies (e.g., Mangun, 1994; Mangun \& Hillyard, 1988; Mangun, Hillyard, \& Luck, 1993; Mulder, Wijers, Brookhuis, Smid, \& Mulder, 1994). These studies have shown greater P1 and N1 amplitudes for stimuli at attended than at unattended locations. Therefore, one may predict larger P1/N1 amplitudes for AR than for IR trials, with control trials in between. In addition, spatial-cuing ERP studies also have reported smaller P300 amplitudes for targets appearing in a validly, rather than an invalidly, cued location (Hillyard, Luck, \& Mangun, 1994; Mangun, 1994). From an activation/inhibition point of view, there- 
fore, one would expect increasing P300 amplitudes in the order of AR, control, and IR.

For the identification task, the activation/inhibition view suggests that priming operates predominantly at later stages of the information-processing chain, such as identification and evaluation of a given stimulus. Hence, due to prime-induced persisting activation/inhibition, less/more event-related activation of neural cell assemblies is necessary to reach an activation threshold required for recognizing the target stimulus (see Houghton \& Tipper, 1994). Given that P300 reflects updating of resources that have been required for the processing of a stimulus (Donchin \& Coles, 1988), P300 amplitude should increase with increasing activation required for processing of the probe display. As a result, also with identification, P300 amplitude should be largest for IR trials, followed by control and AR trials.

Finally, the episodic retrieval view holds that priming effects result from automatic retrieval of prime information if the prime and the probe share one or more stimuli or stimulus locations. Although any aspect of processing of the prime that is or is not appropriate for transfer to processing of the probe may be responsible for behavioral PP or NP effects, respectively (Neill \& Mathis, 1998; Wood \& Milliken, 1998), one interpretation of episodic retrieval deserves particular attention. According to this view, it might be information associated with the prime response that is automatically retrieved on the probe trial whenever the probe shares features with the prime (Milliken, Joordens, Merikle, \& Seiffert, 1998). Since in the AR condition prime and probe responses are the same, correct responses might be activated earlier than in the control condition. This is because response selection in AR trials requires participants only to recognize that the probe display is similar to the prime display, whereas in control trials, selecting a correct response necessitates more profound processing of the probe display. The time course of response activation can be monitored with the so-called lateralized readiness potential (LRP; Gratton, Coles, Sirevaag, Eriksen, \& Donchin, 1988). LRP reflects relative negativity over the contralateral, as compared with the ipsilateral, motor cortex, which monitors the gradual increase in central motor activation of the respective response hand. Given the considerations above, the episodic retrieval view predicts earlier onset of the stimulus-locked LRP for AR than for control trials.

With IR trials, on the other hand, automatic activation of the prime response would result in a tendency to activate the wrong response immediately after probe onset. If prime and probe responses involve different hands, such wrong preactivation should become visible as early LRP positivity, followed by delayed onset of LRP negativity, indicating delayed activation of the correct response. Note that episodic retrieval of prime response information represents the only theoretical approach that would predict wrong LRP preactivation for those IR trials that involve a shift in response hand from the prime to the probe (shift IR trials). On the other hand, earlier LRP onset, relative to the control, can be expected for those IR trials that require same-hand responses to prime and probe displays (noshift IR trials). This is because automatic retrieval of the prime response should result in fast activation of the same hand as that used for the prime response. Since a correct probe response on no-shift IR trials requires the same hand as the preceding prime response, early LRP negativity should be observed. Thus, to test predictions that may be derived from the episodic retrieval view, it is essential to perform separate LRP analyses for shift and no-shift trials. An analogous analysis for LRPs obtained with the localization task is not eligible, due to confounding effects produced by uni- and bilateral probe displays. ${ }^{1}$

On the basis of the assumption that the three major theories of priming might differentially account for identityand location-based priming effects, both types of tasks were employed in the present study. The priming task applied in the present study represents a combination of the location suppression task (Tipper et al., 1990) and the Eriksen flanker task (Eriksen \& Eriksen, 1974). Connelly and Hasher (1993) introduced a similar task, but these authors did not use electrophysiological methods. With the present task, each display consisted of two out of four digits $(1,2,3$, and 4), which were presented at two out of four possible locations corresponding to the ones used in the original location suppression task (Tipper et al., 1990; see Figure 1). The target and distractor stimuli were defined by color. Localization and identification tasks differed only in response cues - that is, the location of the target stimulus in the localization task and the numerical value of the target stimulus in the identification task (see Figure 1).

\section{METHOD}

\section{Participants}

The participants were 10 male and 26 female right-handed undergraduate students ranging in age from 19 to 44 years $(M=$ 22.2 years, $S D=4.6$ years). Their participation served as partial fulfillment of a course requirement. All the participants had normal or corrected-to-normal vision and were naive regarding the purpose of the experiment.

\section{Apparatus and Stimuli}

For presentation of the stimuli, an IBM-compatible computer and a 17-in. SVGA monitor were used. The stimuli were the digits 1, 2, 3, and 4 , presented at two out of four locations on the screen that were underlined by stationary white horizontal bars (see Figure 1). Locations were arranged in two rows and two columns, with the upper two locations slightly farther apart than the bottom two locations. The stimuli appeared in pairs of one red and one blue stimulus. Each stimulus subtended a horizontal visual angle of $0.4^{\circ}$ and a vertical visual angle of $0.6^{\circ}$. The complete display subtended a horizontal visual angle of $3.3^{\circ}$ and a vertical visual angle of $2.2^{\circ}$. Responses were recorded with an accuracy of $\pm 1 \mathrm{msec}$, using a four-button response board, with buttons corresponding by spatial arrangement to the four screen locations.

\section{Procedure}

Identification task. This task consisted of 288 trials divided into two blocks of 144 trials. Prior to the first block, 24 practice trials were presented. Between blocks, there was a break of $30 \mathrm{sec}$. A trial comprised two subsequent displays, the prime and the probe, each containing two of the four digits (see Figure 1). In the AR condition, 


\section{Localization}

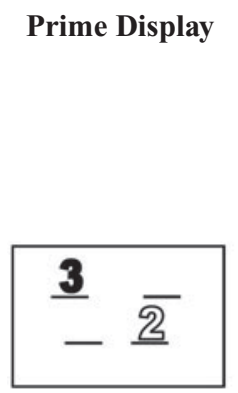

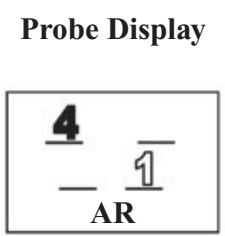
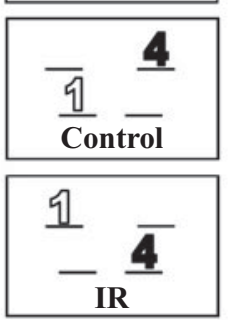

\begin{abstract}
Correct Probe Response
\end{abstract}
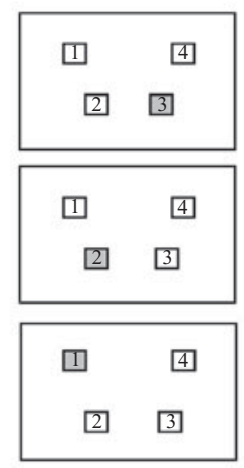

\section{Identification}

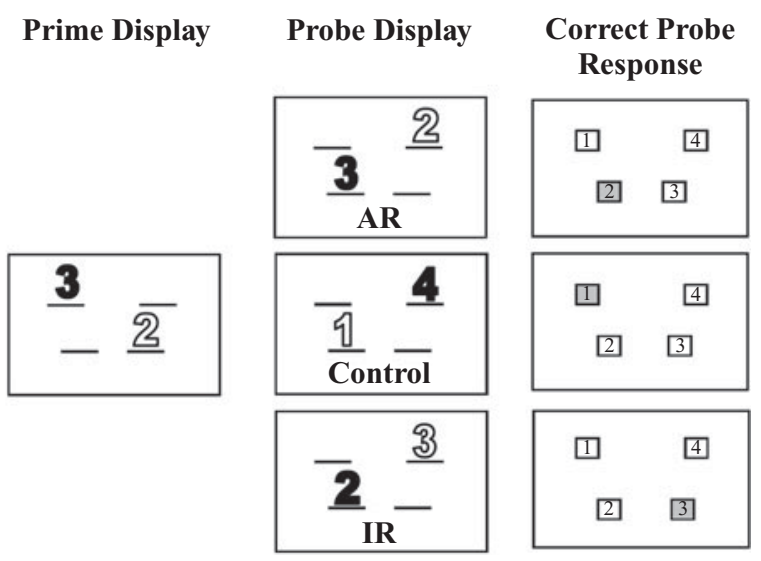

Figure 1. Examples of trials in the localization task (left panel) and in the identification task (right panel). In the leftmost column of each panel a schematic example of a prime display is given. The black digit appeared in blue, and the white digit appeared in red. The middle column depicts examples of attended repetition (AR), control probe, and ignored repetition (IR) displays for the given example of a prime display. In the right column, the gray marked button on the response board represents the correct response to the given probe display (given that target color was red).

the digits presented as target and distractor stimuli were identical in the prime and the probe. Twelve different pairs of target and distractor digits could be obtained from the four digits 1,2, 3, and 4 . Since in AR trials the prime stimuli were repeated on the probe, 12 different AR trials were employed with respect to pairs of stimuli. Similarly, there were 12 different IR trials, since in this condition the prime target and the distractor stimuli were reversed on the probe. The control condition comprised 24 different trials because, for a given prime stimulus, there were two possibilities for assigning the two probe digits to the target and distractor colors. Total numbers of 72 AR trials, 72 IR trials, and 144 control trials were presented, with prime target and distractor locations drawn randomly from the four possible locations. In order to control for location priming, however, in the identification task the probe stimuli always appeared in the two locations not occupied in the prime. AR, IR, and control trials were presented in random order. The participants were instructed to respond as quickly as possible and to avoid making errors by pressing the response button labeled with the digit that was presented in the target color, irrespective of its location.

A trial began with the presentation of the prime, which remained on the screen until a response was made. If the response to the prime was correct, after a response-stimulus interval of $500 \mathrm{msec}$, the probe was presented and remained on the screen until a response was made. In cases of wrong responses to a prime or a probe, an error feedback $(50-\mathrm{msec}$ tone; $1100 \mathrm{~Hz})$ was presented. If there was no response within $1,500 \mathrm{msec}$ after the onset of a prime or a probe, the participant was prompted with a $150-\mathrm{msec}$ tone $(1100 \mathrm{~Hz})$. If a false response was made to the prime or no response was made within 1,500 $\mathrm{msec}$, the trial was canceled and repeated later. False/missing responses to the probe were recorded and classified correspondingly. The trial ended with a correct response to the probe or with the offset of error feedback to the probe. After a 500-msec intertrial interval, the next trial started.

Localization task. As in the identification task, 288 trials were presented, preceded by 24 practice trials. A trial consisted of a prime and a probe, each containing two out of the four digits that were presented at two out of the four locations. In the AR condition, both the probe target and the probe distractor appeared in the same location as that in the prime (see Figure 1). In the IR condition, the locations of target and distractor stimuli were reversed from prime to probe. In the AR, IR, and control conditions, there were 72, 72, and 144 trials, respectively. In order to control for identity priming effects, probe displays always contained digits not presented in the prime. As in the identification task, conditions were randomly intermixed, and the 288 trials were divided into two blocks by breaks of 30 -sec duration. The participants were instructed to respond as quickly and correctly as possible by pressing the response button that corresponded by its position on the response board to the location of the digit that was presented in the target color, irrespective of its numerical value. With respect to trial structure, the localization task was identical to the identification task. Red and blue as target and distractor colors were counterbalanced, respectively, across participants. For a given participant, however, target color was the same in both the identification and the localization tasks.

The participants were seated in a sound-attenuated, dimly lit room $60 \mathrm{~cm}$ in front of a computer monitor. The index and middle fingers of each hand rested on the four response buttons throughout the experiment, so that responding required only movements of the fingers, and not of the hands. Each participant was administered the identification task, followed by the localization task. There was a 10-min break between both parts of the experiment.

As dependent variables, mean RT and percentage of response errors were determined for each of the three experimental conditions (AR, IR, and control). One-way ANOVAs, with experimental conditions as three levels of a repeated measures factor, were computed for RTs and response errors. Hypotheses regarding priming effects were tested by means of planned comparisons with Bonferroni correction.

Electrophysiological recordings. Electrical brain activity was recorded from 11 scalp locations of the 10-20 system (Jasper, 1958) by using an electrode cap (Electrocap Inc.) with sintered $\mathrm{Ag} / \mathrm{AgCl}$ electrodes; the electrode sites were F3, Fz, F4, C3, Cz, C4, P3, Pz, $\mathrm{P} 4, \mathrm{O} 1$, and $\mathrm{O} 2$. The reference was linked mastoids. The vertical EOG was monitored from electrodes positioned below and above the right eye, and the horizontal EOG was monitored from the outer left and right canthi. The EEG was recorded continuously using a 32-channel digital Synamps amplifier and Acquire software (NeuroScan Inc.). Sampling rate was $1000 \mathrm{~Hz}$; bandpass was set to $0.15-70 \mathrm{~Hz}$. The EEG was epoched offline, with epochs ranging from $100 \mathrm{msec}$ before until $1,000 \mathrm{msec}$ after probe display onset. 
Epochs were baseline corrected with respect to the 100-msec interval preceding probe display onset. All the data were screened for artifacts, and contaminated trials were rejected (fewer than $10 \%$ for each participant). EOG correction was performed according to Gratton, Coles, and Donchin (1983). Primary analysis of the data showed that measurements were stable and a sufficient number of trials were obtained, allowing for offline computation of mean ERPs for each participant and each experimental condition. Only epochs involving correct responses were considered. ERP components sensitive to experimental conditions were identified by visual inspection and subsequently subjected to two-way ANOVAs with repeated measures on the factors of condition (AR, control, and IR) and electrode (F3, $\mathrm{Fz}, \mathrm{F} 4, \mathrm{C} 3, \mathrm{Cz}, \mathrm{C} 4, \mathrm{P} 3, \mathrm{Pz}, \mathrm{P} 4, \mathrm{O} 1$, and O2). All effects with more than one degree of freedom were subjected to Greenhouse-Geisser correction.

Separately for participants and experimental conditions, stimuluslocked LRP was determined from averaged waveforms at electrodes C3 and C4. For quantification of LRP, difference waveforms were computed between electrical activity contralateral minus ipsilateral to the correct response and then were averaged across hands (see Coles, 1989). LRP onset was determined as the point in time at which LRP amplitude reached $30 \%$ of its maximum $(30 \%$ relative criterion; see Miller, Patterson, \& Ulrich, 1998). In order to determine LRP onset for 1 participant $i$ in one experimental condition, LRP in this condition was averaged across all the participants except $i$, and then onset detection was performed (jackknife procedure to increase onset reliability; see Miller et al., 1998). Thereafter, mean jackknifed LRP onsets were compared between experimental conditions on the basis of corrected $t$ values according to Miller et al. For assessing LRP preactivation, mean LRP amplitude for each experimental condition was determined from single-subject averaged LRPs within a time window suggested by visual inspection of the waveforms.

\section{RESULTS}

\section{Behavioral Data}

Mean RTs and percentages of wrong responses are shown in Table 1 for AR, IR, and control trials of both priming tasks. Separately for both types of tasks, mean RTs for correct responses to probe displays were subjected to one-way ANOVAs with condition (AR, control, and IR) as a repeated measures factor. For the identification task, a significant effect of condition $[F(2,70)=48.4, p<.001$; $\varepsilon=.66]$ was revealed. Planned comparisons showed that RT for AR trials was significantly decreased, as compared with that for control trials [ 603 and $655 \mathrm{msec} ; t(35)=7.4$, $p<.001$ ], indicating identity-based PP. RTs for control and IR trials did not differ significantly [655 and $656 \mathrm{msec}$; $t(35)=0.2, p=.85]$. Analysis of errors also established a significant effect of condition $[F(2,70)=5.3, p<.01$; $\varepsilon=.95]$. Significantly fewer errors were committed with AR than with control trials $[7.2 \%$ and $8.8 \% ; t(35)=2.5$, $p<.05]$. The difference between control and IR trials was not significant [ $8.8 \%$ and $9.0 \% ; t(35)=0.4, p=.67]$.

For the localization task, an ANOVA yielded a significant effect of condition on RT $[F(2,70)=33.3, p<.001$; $\varepsilon=.68]$. As was revealed by planned comparisons, responses on AR trials were significantly faster than those on control trials [439 and $454 \mathrm{msec} ; t(35)=3.2, p<.01$ ], whereas responses on IR trials were significantly slower, as compared with control trials [ 470 and $454 \mathrm{msec} ; t(35)=$ $7.5, p<.001]$. These findings indicate reliable PP and NP, respectively. A significant effect of condition $[F(2,70)=$ $5.2, p=.01 ; \varepsilon=.91]$ could also be shown for response errors. Although the difference in proportion of errors between AR and control trials failed to become statistically significant [ $4.4 \%$ and $4.6 \% ; t(35)=0.3, p=.77]$, the number of response errors was significantly increased for IR trials, as compared with control trials [6.5\% and $4.6 \%$; $t(35)=2.8, p<.05]$. The latter finding is indicative of location-based NP.

\section{ERP Data}

Visual inspection of waveforms suggested that ERP amplitude, rather than latency, represents a sensitive electrophysiological correlate of behavioral priming effects. For identification, priming-related ERP differences mainly involved P300 amplitude (mean peak latency, $350 \mathrm{msec}$ ), which was smaller for AR trials than for both IR and control trials (see Figure 2). For localization, the amplitudes of the posterior P1 and N1 (mean peak latency, 134 and 176 msec, respectively) were reduced for both AR and IR trials, as compared with control trials. In the P300 time range (mean peak latency, $345 \mathrm{msec}$ ), ERPs were more positive for control trials than for AR trials, with IR trials in between (see Figures 3 and 4). ERP differences observed after mean RT in the slowest experimental condition (470 and $656 \mathrm{msec}$ for the localization and identification tasks, respectively) were excluded from statistical analysis because it is unlikely that these differences reflect processes responsible for behavioral priming effects.

Identification task. In order to investigate the modulation of P300 amplitude by experimental conditions, mean amplitudes obtained under the three experimental conditions within a 100 -msec interval centered around $350 \mathrm{msec}$ were computed and subjected to an ANOVA with condition (AR, control, and IR) and electrode as two

Table 1

Probe Response Times (RTs, in Milliseconds; With Standard Errors of the Means), Priming Effects, and Percentages of Errors as a Function of Task and Experimental Condition

\begin{tabular}{|c|c|c|c|c|c|c|c|c|c|c|c|}
\hline \multirow[b]{3}{*}{ Task } & \multicolumn{4}{|c|}{ AR Condition } & \multicolumn{3}{|c|}{ Control Condition } & \multicolumn{4}{|c|}{ IR Condition } \\
\hline & \multicolumn{2}{|c|}{ RT } & \multirow[b]{2}{*}{ Priming } & \multirow[b]{2}{*}{$\%$ Errors } & \multicolumn{2}{|c|}{ RT } & \multirow[b]{2}{*}{$\%$ Errors } & \multicolumn{2}{|c|}{ RT } & \multirow[b]{2}{*}{ Priming } & \multirow[b]{2}{*}{$\%$ Errors } \\
\hline & $M$ & SEM & & & $M$ & $\overline{S E M}$ & & $M$ & SEM & & \\
\hline Identification & $603^{* * *}$ & 9.0 & 52 & $7.2^{*}$ & 655 & 10.8 & 8.8 & 656 & 11.4 & -1 & 9.0 \\
\hline Localization & $439^{* *}$ & 7.7 & 15 & 4.4 & 454 & 8.5 & 4.6 & $470^{* * *}$ & 8.3 & -16 & $6.5^{*}$ \\
\hline
\end{tabular}

Note-AR, attended repetition; IR, ignored repetition. ${ }^{*} p<.05 .{ }^{* *} p<.01 .{ }^{* * *} p<.001$ (Bonferroni corrected) for the planned comparisons with the control condition. 


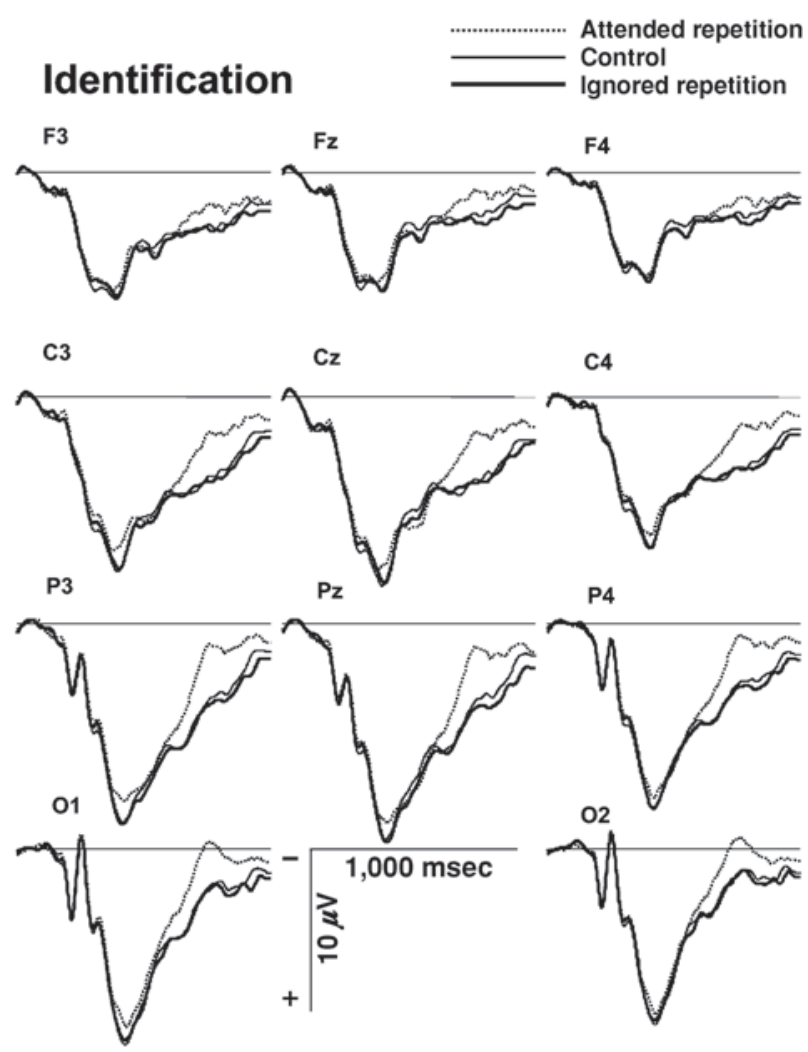

Figure 2. Grand-mean ERP waveforms for probe displays in the identification task as a function of experimental condition. Scale unit, $10 \mu \mathrm{V} / 1,000$ msec. Negativity is plotted upward.

repeated measures factors. Significant main effects of condition $[F(2,70)=6.3, p<.01 ; \varepsilon=.99]$ and electrode $[F(10,350)=28.9, p<.001 ; \varepsilon=.19]$ were revealed. Tukey's HSD test established significantly smaller P300 amplitude for the AR condition than for both the control $(9.7$ and $10.6 \mu \mathrm{V} ; p<.01)$ and the IR $(9.7$ and $10.6 \mu \mathrm{V}$; $p<.01)$ conditions. There was virtually no difference in P300 amplitude between the IR and the control conditions $(p=1.0)$. A significant interaction between condition and electrode $[F(20,700)=2.9, p<.05]$ was found after amplitude rescaling according to McCarthy and Wood (1985). As was indicated by planned comparisons with Bonferroni correction, AR-related P300 amplitude reduction was found to be significant at C3 $(p<.001), \mathrm{P} 3(p<$ $.001), \mathrm{O} 1(p<.01), \mathrm{Cz}(p<.05), \mathrm{Pz}(p<.05), \mathrm{C} 4(p<$ $.05)$, and P4 ( $p<.05$; see Figure 2$)$. Moreover, P300 amplitude was significantly smaller for AR than for IR at P3 $(p<.001), \mathrm{O} 1(p<.01), \mathrm{Cz}(p<.05), \mathrm{Pz}(p<.01)$, and $\mathrm{P} 4(p<.05)$. At neither electrode was a significant difference found between the IR and the control conditions (all $p$ values $>.50)$.

Localization task. Decreased P1-N1 peak-to-peak amplitude, elicited by probe displays, was observed for both AR and IR trials, as compared with control trials (see Figure 4). Peaks were detected within individual time windows ranging from 120 to $150 \mathrm{msec}(\mathrm{P} 1)$ and from 150 to
$200 \mathrm{msec}(\mathrm{N} 1)$. Mean peak latencies across experimental conditions were 134 and $179 \mathrm{msec}$ for P1 and N1, respectively. Peak-to-peak amplitude ${ }^{2}$ was subjected to a $3 \times$ 11 ANOVA with factors of condition (AR, control, and IR) and electrode. There was no significant main effect of condition $[F(2,70)=1.2, p=.31 ; \varepsilon=.81]$. A significant main effect of electrode $[F(10,350)=98.6, p<.001 ; \varepsilon=$ .19] indicated that, with visual stimuli, P1-N1 amplitudes were most pronounced over occipital areas. After amplitude rescaling across conditions, a significant interaction between condition and electrode $[F(10,350)=2.4, p<$ $.05 ; \varepsilon=.27$ ] could be established. More pronounced $\mathrm{P} 1-$ $\mathrm{N} 1$ peak-to-peak differences were revealed by $t$ tests for control than for AR trials at P3 [3.0 and $2.0 \mu \mathrm{V} ; t(35)=$ 2.3, $p<.05]$ and $\mathrm{O} 1$ [6.3 and $5.1 \mu \mathrm{V} ; t(35)=2.4, p<$ .05]. P1-N1 amplitude was significantly larger for control than for IR trials at $\mathrm{Pz}[2.0$ and $1.0 \mu \mathrm{V} ; t(35)=2.8, p<$ $.01]$ and $\mathrm{O} 2$ [5.6 and $4.8 \mu \mathrm{V} ; t(35)=2.2, p<.05$; see Figure 4]. At the remaining electrode sites, no significant differences were observed. Furthermore, there were no significant differences between AR and IR trials at any of the 11 electrode sites.

Within the P300 time range, ERPs were less positive for AR than for control trials, with IR trials in between (see Figures 3 and 4). Analogously to the P300 analysis for the identification task, mean amplitude between 300 and $400 \mathrm{msec}$ was calculated and subjected to an

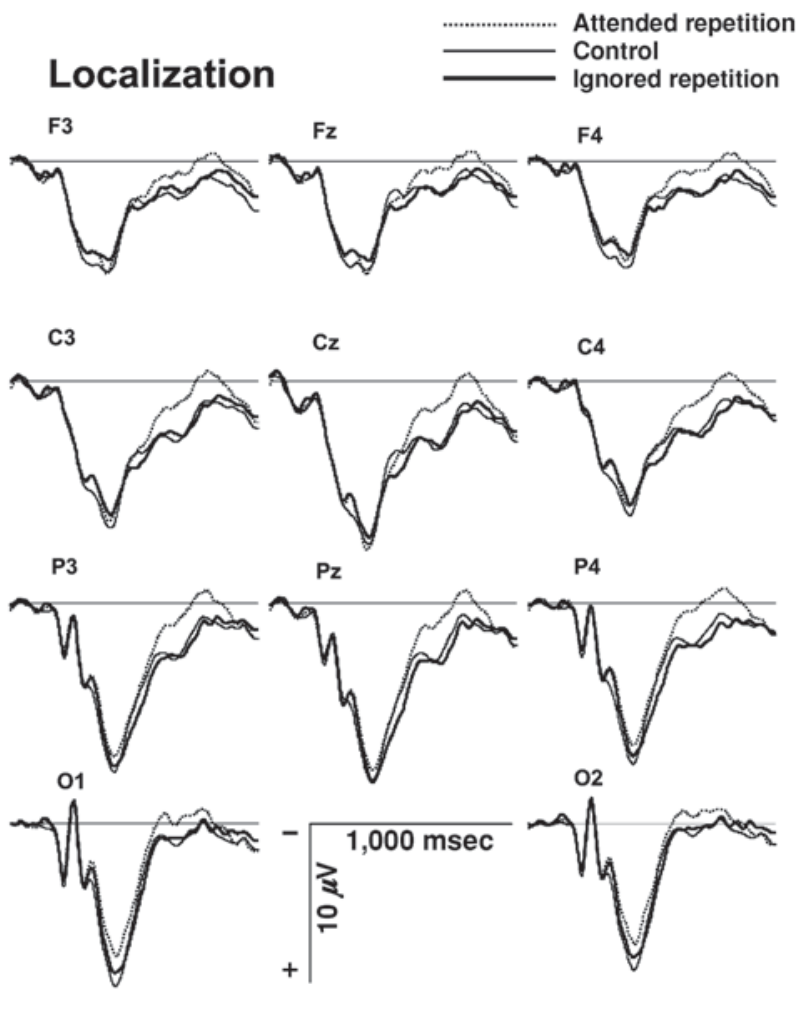

Figure 3. Grand-mean ERP waveforms for probe displays in the localization task as a function of experimental condition. Scale unit, $10 \mu \mathrm{V} / 1,000$ msec. Negativity is plotted upward. 


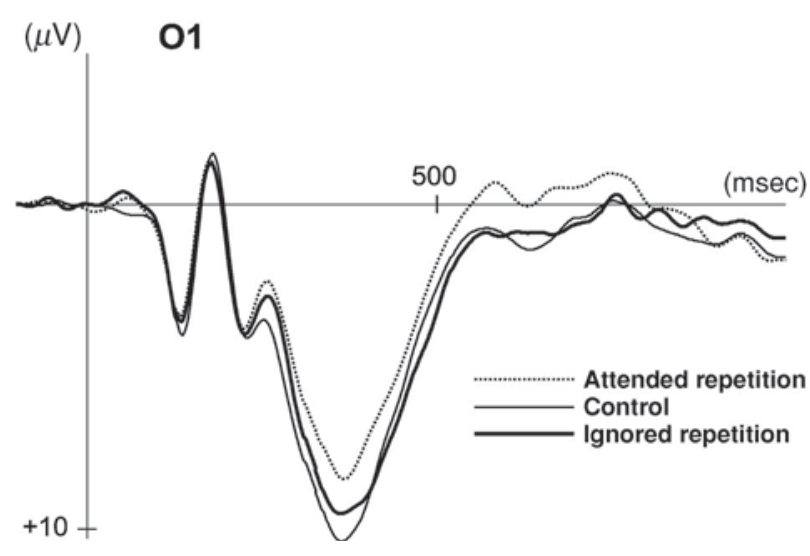

Figure 4. Grand-mean left-occipital ERP waveforms for probe displays in the localization task as a function of experimental condition. Scale unit, $10 \mu V / 1,000$ msec. Negativity is plotted upward.

ANOVA with repeated measures on condition (AR, control, and IR) and electrode. Significant main effects of condition $[F(2,70)=4.0, p<.05 ; \varepsilon=.80]$ and electrode $[F(10,350)=13.7, p<.001 ; \varepsilon=.18]$ were obtained. Tukey's HSD tests revealed that mean amplitude was less positive for AR than for control trials $(8.2$ and $9.1 \mu \mathrm{V} ; p<$ $.05)$. Differences between IR and control trials $(8.5$ and $9.1 \mu \mathrm{V} ; p=.08)$ and $\mathrm{AR}$ and IR trials $(8.2$ and $8.5 \mu \mathrm{V}$; $p=.72$ ) failed to become statistically significant. There was a significant interaction of condition and electrode after amplitude rescaling $[F(20,700)=4.7, p<.001 ; \varepsilon=$ .22]. Bonferroni-corrected planned comparisons yielded significant priming-related differences in ERP positivity, which were restricted to lateral posterior sites. At lateral parietal electrodes P3 and P4, P300 amplitude in the AR condition was significantly smaller than that in the control condition [P3, 9.7 and $10.8 \mu \mathrm{V} ; t(35)=3.7, p<.01 ; \mathrm{P} 4$, 8.8 and $10.1 \mu \mathrm{V} ; p<.001]$, whereas the differences between the IR and the control conditions and between the
IR and the AR conditions did not become significant (all $p$ values $>.20$ ). At $\mathrm{O} 1, \mathrm{P} 300$ amplitude was largest for the control condition, followed by the IR and AR conditions $(10.1,9.3$, and $8.0 \mu \mathrm{V})$; differences between all three conditions were significant [AR and control, $t(35)=6.1$, $p<.001 ;$ IR and control, $t(35)=3.0, p=.01 ; \mathrm{AR}$ and IR, $t(35)=3.4, p<.01]$. Similarly, P300 amplitude at $\mathrm{O} 2$ was significantly smaller for the AR condition than for the control condition $[7.2$ and $9.0 \mu \mathrm{V} ; t(35)=5.5, p<$ $.001]$ and for the AR condition than for the IR condition [7.2 and $8.4 \mu \mathrm{V} ; t(35)=3.3, p<.01]$. Also, the difference between the IR and the control conditions became significant [8.4 and $9.0 \mu \mathrm{V} ; t(35)=2.5, p<.05$ ] .

\section{LRP Data}

Identification task. The LRPs accompanying probe responses were computed from electrodes $\mathrm{C} 3$ and $\mathrm{C} 4$. As can be seen in Figure 5, pronounced LRP negativity developed after an initial phase of zero baseline. At the end of the analyzed 1,000-msec epoch, substantial LRP negativity was still present. Figure 6 shows mean LRPs in the identification task as a function of experimental conditions. IR and control trials were subdivided into shift and no-shift trials. This did not apply for AR trials, since all the AR trials were no-shift trials. Typical LRP waveforms involving pronounced negativity developed only on shift IR and shift control trials. On AR, no-shift IR, and no-shift control trials, LRP negativity was almost completely absent. Rather, on the latter three types of trials, LRP was temporarily positive going, an effect that started within the first $200 \mathrm{msec}$ and peaked at around $250 \mathrm{msec}$. The subsequent negative-going portion of these atypical LRP waveforms peaked at around $500 \mathrm{msec}$ but only just crossed zero. Negative peaks, however, occurred at about the same time as in shift IR and shift control trials, both of which elicited typical LRPs. This pattern of results suggests small LRPs for AR, no-shift IR, and no-shift control trials, riding on a positive shift that had started earlier (see Figure 6).

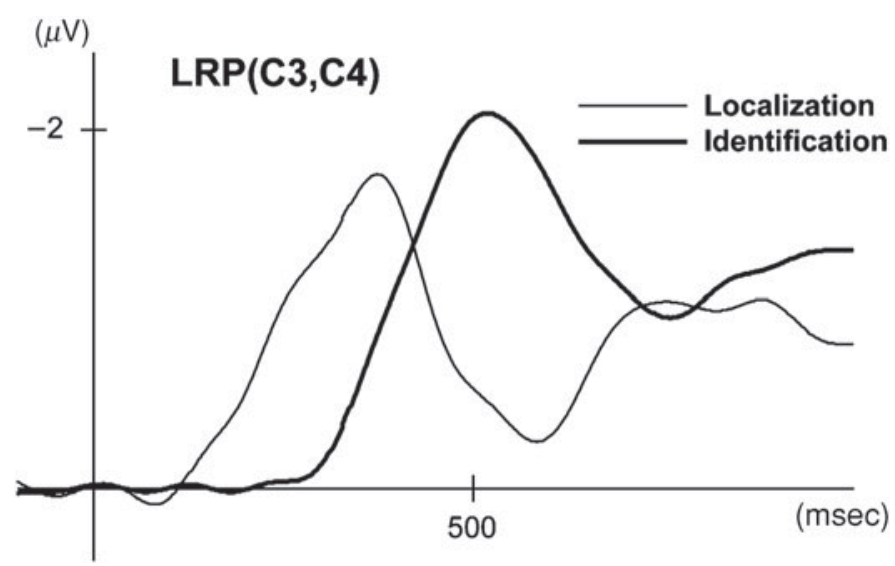

Figure 5. Grand-mean lateralized readiness potentials (LRPs) accompanying probe responses in the identification (bold line) and localization tasks. Scale unit, $2 \mu \mathrm{V} / 1,000 \mathrm{msec}$. Negativity is plotted upward. 


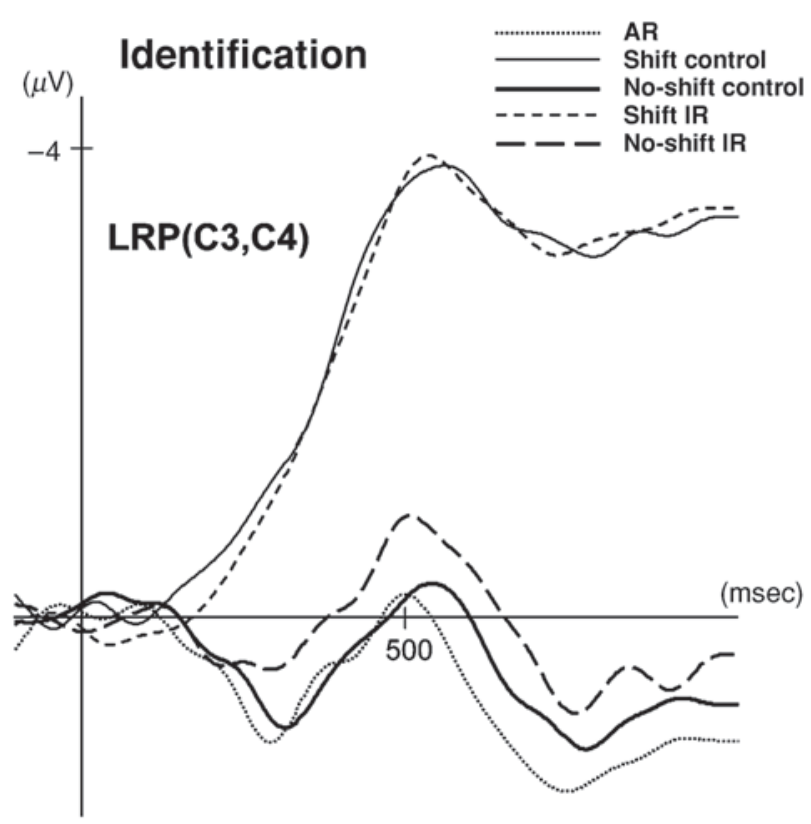

Figure 6. Mean lateralized readiness potentials (LRPs) accompanying probe responses in the identification task as a function of experimental condition, which was further classified into shift trials and no-shift trials. AR, attended repetition; IR, ignored repetition. Scale unit, $4 \mu \mathrm{V} / 1,000$ msec. Negativity is plotted upward.

In order to investigate this pattern of results, late LRP negativity in the overall waveform (see Figure 5) was quantified as mean LRP amplitude within the last $100 \mathrm{msec}$ of each epoch (900-1,000 msec). Mean late LRP negativity across all three experimental conditions was $-1.3 \mu \mathrm{V}$, which was different from zero at the $1 \%$ level of significance (the $99.9 \%$ confidence interval ranged from -0.8 to $-1.7 \mu \mathrm{V})$. This finding clearly indicated that, at the onset of the subsequent display presentation, the hand used for the last response was still in a state of activation.

Mean early LRP amplitude was computed within a time window from 250 to $300 \mathrm{msec}$ where positive preactivation was highest. A one-way ANOVA was performed involving repeated measures on condition (AR, shift control, no-shift control, shift IR, and no-shift IR). The effect of condition was highly significant $[F(4,140)=21.0, p<$ $.001 ; \varepsilon=.66]$. Planned comparisons revealed significant differences in early LRP amplitude between AR trials $(0.9 \mu \mathrm{V})$ and both shift control trials $[-1.3 \mu \mathrm{V} ; t(35)=$ $6.4, p<.001]$ and shift IR trials $[-1.1 \mu \mathrm{V} ; t(35)=5.2$, $p<.001]$. Also, the difference between shift and no-shift control trials was significant $[-1.3$ and $0.9 \mu \mathrm{V} ; t(35)=$ $6.1, p<.001]$, as was the difference between no-shift control and shift IR trials $[0.9$ and $-1.1 \mu \mathrm{V} ; t(35)=5.3, p<$ $.001]$. Finally, on no-shift IR trials, significantly more pronounced early LRP positivity was observed than on shift control trials $[0.4$ and $-1.3 \mu \mathrm{V} ; t(35)=4.9, p<.001]$ and shift IR trials [0.4 and $-1.1 \mu \mathrm{V} ; t(35)=3.7, p<.01]$. There was no significant difference in LRP preactivation between shift IR and shift control trials $(p>.50)$ and be- tween AR, no-shift control, and no-shift IR trials (all $p$ values $>.10$; see Figure 6).

LRP onsets were compared only between shift IR and shift control trials, since for the remaining types of trials, LRP onset estimates could not be validly determined, due to predominantly positive amplitudes. As was suggested previously (Miller et al., 1998), jackknifed LRP onset scores were determined separately for the control and IR conditions, using a 30\% relative criterion. Mean jackknifed LRP onsets were 305 and $293 \mathrm{msec}$ for shift IR and control trials [corrected $t(35)=1.1, p=.28$; see Figure 6].

To determine whether the different LRP patterns for shift and no-shift trials were accompanied by different behavioral NP effects, mean RTs and percentages of errors for IR and control trials were computed separately for shift and no-shift trials. RTs did not differ between IR and control on both shift trials [655 and $655 \mathrm{msec} ; t(35)=$ $0.01, p=.99$ ] and no-shift trials [656 and $654 \mathrm{msec}$; $t(35)=0.43, p=.67]$. This outcome indicates absence of behavioral NP effects. Also with regard to percentages of errors, the difference between IR and control was significant neither for shift trials $[8.0 \%$ and $8.6 \% ; t(35)=0.7$, $p=.47]$ nor for no-shift trials $[10.8 \%$ and $9.3 \% ; t(35)=$ $1.3, p=.21]$.

Localization task. Figure 7 depicts LRP waveforms recorded from $\mathrm{C} 3$ and $\mathrm{C} 4$ separately for location-based AR, IR, and control conditions (a distinction between shift trials and no-shift trials cannot be expected to yield meaningful results; see the introduction). As for the identification task, pronounced LRP negativity was observed for both IR and control trials, but not for AR trials. As with the identity-based AR condition, also for location-based AR trials there was early LRP positivity, followed only by weak LRP negativity. This notion is supported by the fact that grand-average LRP peak latencies for AR, control, and IR trials (360, 374 and $386 \mathrm{msec}$, respectively) corresponded well to mean RTs $(439,454$, and $470 \mathrm{msec}$, respectively). As with the identification task, when the data were collapsed across conditions, there was late LRP negativity, lasting until the next display presentation (see Figure 5).

In a first step, late LRP negativity was investigated within the last $100 \mathrm{msec}$ of the analyzed epoch (900$1,000 \mathrm{msec}$ ). Mean late LRP amplitude across experimental conditions was $-0.9 \mu \mathrm{V}$, which was different from zero at the $1 \%$ level of significance (the $99.9 \%$ confidence interval ranged from $-0.3 \mu \mathrm{V}$ to $-1.5 \mu \mathrm{V}$; see Figure 5). Positive preactivation on AR trials was determined as mean LRP amplitude within a time window ranging from 120 to $180 \mathrm{msec}$. An ANOVA with repeated measures on condition (AR, control, and IR) yielded a significant main effect $[F(2,70)=8.6, p=.001 ; \varepsilon=.78]$. Planned comparisons revealed that early LRP was reliably more positive going for AR trials $(0.2 \mu \mathrm{V})$ than for control trials $[-0.5 \mu \mathrm{V} ; t(35)=3.7, p=.001]$ and IR trials $[-0.3 \mu \mathrm{V}$; $t(35)=2.5, p<.05]$. There was no significant difference in LRP preactivation between IR and control trials $[-0.3$ 


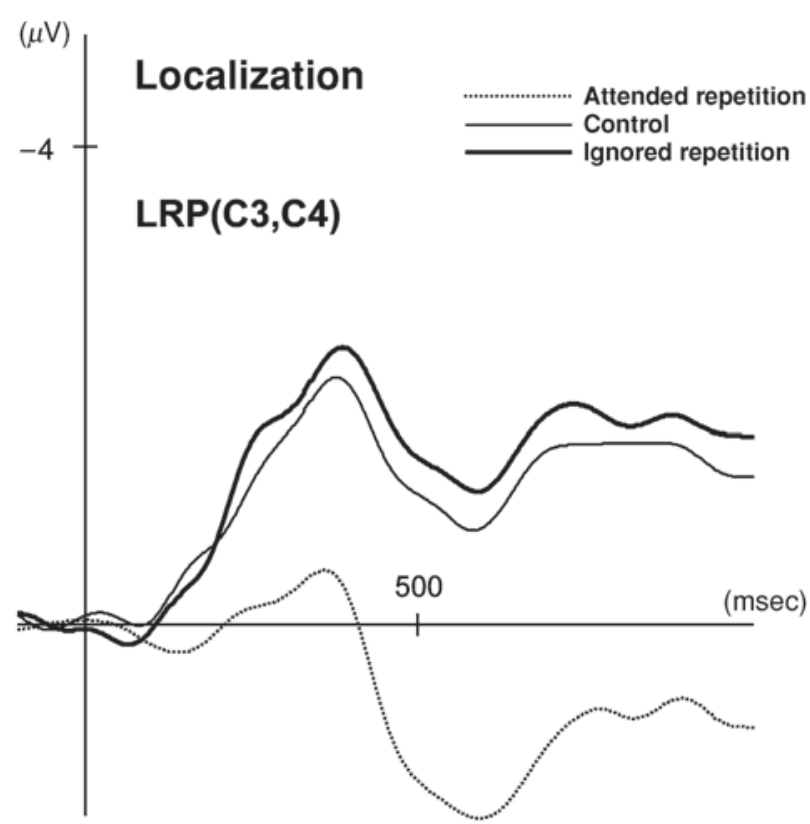

Figure 7. Mean lateralized readiness potentials (LRPs) accompanying probe responses in the localization task as a function of experimental condition. Scale unit, $4 \mu \mathrm{V} / 1,000 \mathrm{msec}$. Negativity is plotted upward.

and $-0.5 \mu \mathrm{V} ; t(35)=1.2, p=.23]$. Mean jackknifed LRP onsets according to the $30 \%$ relative criterion were 190 and $195 \mathrm{msec}$ for control and IR trials, respectively $[t(35)=0.6, p=.59$; see Figure 7$]$.

\section{DISCUSSION}

With the use of ERP recordings, the aim of the present study was to investigate brain correlates of PP and NP obtained by means of a localization and an identification task. Since ERPs represent more direct reflections of the time course of information processing than do RTs, we expected to identify ERP antecedents of behavioral priming effects that might help to evaluate the validity of the three major theoretical accounts of priming effects-feature match/mismatch theory, activation/inhibition theory, and episodic retrieval theory.

With the exception of identity-based NP, all expected behavioral priming effects could be established. The failure to observe identity-based NP may be due to the fact that the target and the distractor digits were presented at relatively distant locations (see Figure 1). This way, the localization and identification tasks could be made as similar as possible. However, identity-based NP may necessitate a highly salient prime distractor stimulus, which could perhaps be best achieved with spatially close or overlapping target and distractor stimuli (e.g., Fox, 1994; see also Fox, 1995a).

For the observed behavioral priming effects, specific electrophysiological ERP correlates of priming could be revealed. In the identification task, faster responding to AR probes was accompanied by reduced P300 amplitude over central-to-occipital electrode sites. Also for AR trials, an atypical LRP pattern was observed that involved weak LRP negativity riding on early LRP positivity. Although there was no behavioral NP effect, analysis of IR and control trials revealed an intriguing pattern of LRP waveforms. In both no-shift IR and no-shift control trials, the same atypical pattern of early LRP positivity, followed by weak negativity, was observed as in AR trials, whereas typical (negative-only) LRPs were obtained with shift IR and shift control trials.

\section{Positive Identity Priming and P300 Amplitude}

P300 amplitude is commonly considered as reflecting the amount of resources allocated to stimulus evaluation or the effort required for integrating recent stimulation into a model of the environment (Donchin \& Coles, 1988). From this perspective, the present ERP data suggest that identity-based PP may be produced by facilitation of stimulus evaluation in the AR condition. Thus far, our data are consistent with the activation view of PP (Houghton \& Tipper, 1994; Morton, 1969, 1979; Tipper \& Cranston, 1985). According to this approach, persisting activation of stimulus representations from the prime results in less effort to activate these stimulus representations a second time. In other words, less activation of corresponding neuronal assemblies is needed in order to reach an activation threshold required for successful stimulus evaluation. This might be reflected by smaller P300 amplitude as an index of activity evoked by the AR probe display. The finding of reduced activity as a brain correlate of identity-based PP is well in line with imaging studies showing that the repetition of stimuli was generally accompanied by reduced activity in lateral posterior areas (for a review, see Henson, 2003).

It is important to note that the episodic retrieval view also can account for the reduction of P300 amplitude as a correlate of identity-based PP. From this perspective, participants are assumed to quickly recognize that probe displays in the present AR condition contain the same stimuli as those in the preceding prime display. Identical probe stimuli may serve as a cue to automatically retrieve the prime response, which then can be executed without the need to exhaustively analyze the probe display (see Milliken et al., 1998; Neill et al., 1992). Alternatively, prime information other than that regarding the correct response may be retrieved from the prime and may facilitate probe processing (e.g., Neill \& Mathis, 1998). Thus, within the framework of the episodic retrieval view, the reduced P300 amplitude observed with AR trials, relative to control trials, reflects the fact that, in order to select a correct response, AR probe displays have to be analyzed in less detail than do probe displays on control trials. On the other hand, the activation view assumes qualitatively identical information processing for both AR and control trials, which, however, is predicted to be less effortful in the former condition. Obviously, it is not possible to decide between these two theoretical accounts on the basis of our P300 data. 
Ratcliff and McKoon's (1997) counter model, as well as the ROUSE (Huber, Shiffrin, Lyle, \& Ruys, 2001) and REMI (Schooler, Shiffrin, \& Raaijmakers, 2001) models of implicit priming, have recently received much attention. All three models explain repetition priming in terms of a decision bias, rather than in terms of encoding facilitation. Since the matching of information against a prior processing episode is emphasized, these models can be considered variations of the episodic retrieval view. Most relevant to the present study may be the ROUSE (responding optimally with $u$ nknown sources of evidence; Huber et al., 2001) model of short-term priming, although it has been developed on the basis of a largely different task. With this task, one word is briefly flashed and then has to be correctly selected from two alternative words. Interestingly, the probability of correct responses is larger when the flashed word has been passively studied immediately before than when it has been actively studied. This finding is at variance with an encoding-facilitation/persistentactivation account of repetition priming. The ROUSE model rather assumes that participants compensate for source confusion (i.e., certain perceptual features may be "on" after probe onset, due to either probe or prime presentations) by actively attenuating the processing of prime features, an implicit strategy referred to as discounting. The effect of active/passive prime processing can be explained by stronger discounting after more profound prime analysis.

The present data from both identification and localization tasks do not support the notion of a discounting strategy, because strong PP was found for RTs, although ROUSE predicts strong discounting. To reiterate, in the present tasks, primes were actively processed to select a response. An aim of future research should be to directly compare PP effects from actively and passively processed prime displays, to determine whether or not discounting is operative also in traditional RT priming tasks.

\section{LRP Data in the Identification Task}

In several respects, the LRP that monitors the time course of hand-specific motor activation provided important additional evidence that may help to expand our understanding of the processes involved in performing the present two tasks. LRP analysis allowed for a direct test of several predictions derived from a variant of the episodic retrieval view (e.g., Milliken et al., 1998). Retrieval of the prime response occurs because, in the probe display, specific stimuli are repeated from the prime. If so, early activation of the correct response hand should be observed in terms of early LRP negativity on AR trials before LRP negativity becomes apparent on control trials, because, in the latter condition, no stimuli are repeated from the prime and activation of the correct response cannot occur until a more profound analysis of the probe display has been completed. By contrast, for IR trials, early LRP positivity should be observed, which reflects temporary activation of a wrong response. However, as was outlined above in the introduction, this is true only for IR trials involving a shift in response hand from the prime to the probe (shift
IR trials). On no-shift IR trials, the target and the distractor were color reversed from the prime to the probe but still required same-hand responses. Hence, retrieval of the prime response should result in early activation of the correct response hand and, consequently, should evoke early LRP negativity similar to that expected for AR trials.

Proceeding from these considerations, the present LRP findings are at variance with a retrieval-of-response interpretation of the episodic retrieval view. There was neither early LRP positivity on shift-IR trials nor early LRP negativity on AR and no-shift IR trials. Instead, both AR and no-shift IR trials elicited significant early LRP positivity, followed by the development of much reduced LRP negativity for both types of trials. Also relevant to the interpretation of the present LRP results is the finding of the same atypical LRP pattern showing early LRP positivity for no-shift control trials (see Figure 6). On these trials, no probe stimulus was repeated from the prime, and according to the episodic retrieval view, no prime information should have been retrieved. The finding of response activation starting $150 \mathrm{msec}$ after probe display onset and, thus, clearly preceding completion of a profound analysis is hard to explain within the framework of episodic retrieval.

In order to better understand the nature of the cognitive processes underlying this unexpected pattern of LRP data, several observations should be emphasized. First, in the grand-average LRP computed across all the experimental conditions, substantial negativity was observed at the time of presentation of the subsequent display. This late LRP negativity was not directly associated with priming but, rather, reflected a general, implicit or explicit strategy of the participants to always maintain activation of the most recently used response hand until the next display was presented. Second, all the experimental conditions involving same-hand responses to prime and probe displays (AR, no-shift control, and no-shift IR) showed atypical LRP patterns with no substantial LRP negativity, whereas all the conditions requiring different hands for prime and probe responses elicited substantial LRP negativity. In combination with the finding of persisting LRP activity until the onset of the subsequent display, this suggests a benefit from prime-induced motor activation whenever the probe display requires a same-hand response. In other words, to successfully execute the correct response, less additional motor activation is necessary, which, in turn, is reflected by only weak LRP negativity.

Within the context of the LRP literature, the idea of persisting motor activation from a prior response is not entirely new. Employing a two-choice RT task, Jentzsch and Sommer (2002) observed monotonically increasing magnitude of long-term LRP computed across several trials as a function of number of repetitions of the same response hand. Moreover, as the amplitude of residual LRP traces increased, the LRP amplitude of the current response monotonically decreased. These LRP effects were accompanied by decreasing RT as a function of subsequent hand repetitions. Nevertheless, evidence for residual LRP negativity with the present task represents a novel and 
intriguing finding, since a four-choice RT task was employed, with two keys assigned to two different fingers of each hand. Thus, in the present study, keeping the motor program in a state of activation after completion of the prime response represented a less useful strategy, because the probability that this specific program is required for a correct probe response was only .25 , as opposed to .50 with the two-choice task applied by Jentzsch and Sommer. Therefore, it is interesting to note that persisting motor activation from the prime was observed with the present four-choice RT task. Another finding not yet reported in the LRP literature was the counterintuitive temporary LRP positivity early after onset of displays that required a response that was still highly activated.

Given this background, the unexpected finding of early LRP positivity with AR, no-shift IR, and no-shift control trials can be explained as follows. Rather than indicating preparation of the wrong response side (as early LRP positivity is often understood; e.g., Valle-Inclan, 1996), the observed LRP effect may reflect temporary inhibition of a response tendency that is already in a state of high activation (see Smid, Fiedler, \& Heinze, 2000). ${ }^{3}$ As soon as analysis of the probe display has verified that the correct response involves the same hand as the immediately preceding response, temporary inhibition of the persistent hand-specific activation will be removed. Thus, almost no additional hand-specific activation is necessary for response execution. This assumption receives support from the observation that in all three experimental conditions involving repetition of response hand (AR, no-shift control, and no-shift IR), LRP negativity did not markedly exceed initial baseline levels.

One could argue that early LRP positivity in those experimental conditions involving repetition of the response hand represents a passive, rather than an active, process. Motor activation persisting from the prime may decay as a function of time. This scenario, however, does not appear very likely, because, after a temporary decrease following response execution, LRP negativity again increased as the next display presentation approached (see Figure 5). Furthermore, from $100 \mathrm{msec}$ before until $150 \mathrm{msec}$ after probe display onset, LRP was generally flat and close to zero (see Figure 6), which contradicts the assumption that early LRP positivity during probe processing was produced by a gradual weakening of prime-induced LRP negativity.

Active inhibition of an already activated response could be useful for avoiding premature repetition of the prime response before probe display analysis is completed. Additional hand-specific motor activation resulting from a preliminary probe analysis might excite the still activated prime response beyond the threshold for execution before the correct finger has been determined. This would result in a wrong response whenever the correct probe response required the same hand as the prime response but a different finger. For AR trials, on the other hand, sufficiently deep analysis reveals that the correct probe response is, indeed, the same as the preceding prime response, and inhibition of the motor response can simply be removed. In this case, almost no additional motor activation seems to be necessary to repeat the prime response, as is indicated by LRP negativity's barely exceeding baseline.

However, LRP negativity was comparably weak for noshift control trials and no-shift IR trials, although on these trials the prime response could not simply be repeated. Instead, another finger of the same hand had to be selected for the correct probe response. It appears that persisting motor activation from the last response was utilized by the participants in a highly selective manner that differentiated hand-specific activation from activation related to specific finger movements. On AR trials, after removal of inhibition, the complete motor program from the prime could be repeated without further specifications. With no-shift IR and no-shift control trials, the correct probe response could benefit only from hand-specific motor activation that persisted from the prime, whereas finger selection still required additional motor activation. Activation related to finger selection, however, cannot become evident in LRP waveforms, which, consequently, showed a weak negativity almost identical to that for AR trials. Finally, shift IR and shift control trials, on which selection of the correct probe response also necessitated activation of a different hand, produced full-scale LRP negativity, because no hand-specific motor activation persisting from the preceding prime could be utilized. It should be noted that persistence of the complete motor program of the prime response, rather than merely persisting hand-specific activation, seemed to be responsible for behavioral facilitation with AR trials, because identical RTs were observed for shift and no-shift control trials, as well as for shift and no-shift IR trials, despite differences in persisting hand-specific activation between these experimental conditions.

\section{Positive and Negative Location Priming and P1/N1 Amplitudes}

In the localization task, the earliest identified ERP priming correlate was a reduction of posterior $\mathrm{P} 1 / \mathrm{N} 1$ amplitude for both AR and IR trials, as compared with control trials, whereas there was no AR-IR difference. Furthermore, P300 amplitude at posterior electrode sites differed significantly between all three experimental conditions, with the smallest amplitude for the AR condition, followed by the IR and control conditions. Location-based PP was accompanied by an atypical LRP waveform for AR trials similar to the one observed with identity-based AR trials. In both the location-based IR and the control conditions, typical LRPs developed that differed neither with respect to preactivation nor with respect to onset latency. However, when the data were collapsed across experimental conditions, a substantial late LRP negativity was found that was similar to the one observed with the identification task.

At least two different explanations may account for the amplitude reduction of early sensory components $\mathrm{P} 1$ and $\mathrm{N} 1$ with both AR and IR trials. The first explanation refers to inhibition of return (IOR), a mechanism associated with selective attention. IOR describes a tendency to inhibit 
orienting toward visual locations that have recently been attended (Posner \& Cohen, 1984). To demonstrate IOR, at least $300 \mathrm{msec}$ must have elapsed between the time at which attention was first captured by a certain location and the appearance of another stimulus in the same location (Posner \& Cohen, 1984). Furthermore, processing of the second stimulus suffers from IOR only if attention has already been disengaged from this location after first attending to it. In the present study, the interval between the prime and the probe displays was $500 \mathrm{msec}$. Because with AR and IR trials, probe stimuli appeared in the same locations as the prime stimuli, IOR was likely to be active under these conditions. Therefore, at the time of probe onset, attention was less likely allocated to the locations that were occupied on the prime display than to those that were empty. This means that probe stimuli in the control condition appeared with a higher probability in locations that had already been attended to, as compared with probe stimuli in the AR and IR conditions. From this perspective, the present finding of reduced $\mathrm{P} 1 / \mathrm{N} 1$ amplitude for both AR and IR trials is well in line with the literature on spatial selective attention effects on ERPs. Numerous studies have shown larger P1 and N1 amplitudes when visual stimuli were presented in attended, as compared with unattended, locations (e.g., Eason, Harter, \& White, 1969; Eimer \& Schröger, 1995; Luck, Heinze, Mangun, $\&$ Hillyard, 1990). The IOR explanation of our data is consistent with a recent ERP study (McDonald, Ward, \& Kiehl, 1999) reporting reduced P1 amplitude accompanying behavioral IOR.

Although IOR may easily account for the present finding of behavioral NP observed with IR trials (see also Christie \& Klein, 2001; Milliken et al., 2000), it is more difficult to understand why IOR should also be effective in the AR condition in which behavioral PP was observed. As a solution to this apparent inconsistency, several authors have put forward the idea of opponent facilitatory and inhibitory processes involved in location-based priming (Christie \& Klein, 2001; Gibbons \& Rammsayer, 2004; Milliken et al., 2000; Pratt \& Abrams, 1999). Milliken et al. (2000) showed that repeating nonspatial stimulus features in a given location produces an RT advantage that counteracts IOR-induced slowing of RT. Gibbons and Rammsayer compared spatial AR effects in conditions in which disengagement of attention from the prime target location was more or less likely. Significant PP was observed with predictably long probe displays - that is, when there was no need to quickly disengage attention from the prime target location in order to be prepared for a probe target that could appear in any of the four possible locations. On the other hand, in conditions with no prior information about probe duration, the participants were assumed to expect brief probe display durations and to disengage attention from the prime target location. Under these conditions, IOR was likely to become effective. Consistent with this idea, no significant PP effect was observed, and sometimes the RT on AR trials was even longer than the RT on control trials, whenever a brief probe display was expected. In addition, our present ERP data provided con- verging electrophysiological evidence for the existence of IOR during cognitive processing of AR trials. Nevertheless, the finding of a reliable behavioral PP effect for AR trials, despite the counteracting IOR effect, points to a strong facilitatory influence that became effective under the AR condition (see below).

There is at least one alternative, neurophysiological explanation for the P1/N1 reduction observed with AR and IR trials. When the probe display was presented, neurons involved in spatially selective information processing could still be in a state of refractoriness (see Eimer, 1994). This could result in reduced P1/N1 amplitude, since the same two locations were activated by both the prime and the probe displays. However, sensory refractoriness should become most relevant when identical stimuli are repeated in the same locations. This was not true for the present localization task, where generally, two digits different in shape from those in the preceding prime were presented on the probe (see Figure 1). Furthermore, in the AR condition, same-color stimuli were repeated in certain locations, as opposed to different-color stimuli in the IR condition. Therefore, a P1/N1 reduction that would exclusively reflect sensory refractoriness should have been more pronounced for AR than for IR trials. This pattern of results, however, was not observed.

Irrespective of whether the P1/N1 amplitude effect was due to sensory refractoriness or IOR, this effect is at variance with feature match/mismatch theory as it is commonly understood. If the opponent behavioral effects with AR and IR trials were exclusively due to facilitation and inhibition of early sensory processing, opponent early ERP differences from the control for AR and IR trials would be the expected outcome. Early sensory ERP effects, however, were the same for AR and IR trials. A cautionary note concerns the fact that feature match/mismatch theory in a more general meaning must not necessarily locate behavioral priming effects in sensory analysis. According to Park and Kanwisher (1994), it remains unclear whether match/mismatch processes also occur between abstract stimulus features, such as symbol identity or response category, which may exist only within the context of a particular task.

\section{Positive and Negative Location Priming and P300 Amplitude}

Location-based experimental conditions also affected P300 amplitude. The observed pattern of P300 amplitudes did not support the activation/inhibition view. Within this framework, P300 amplitude should have increased in the order of AR, control, and IR, reflecting increasing effort with the evaluation of stimuli appearing in locations that were subject to persisting activation, no persisting activation, or persisting inhibition, respectively. However, at posterior electrode sites, P300 amplitudes for both AR and IR trials were significantly smaller than those for control trials. This pattern of results was not compatible with the notion that differential efforts for stimulus evaluation would account for the opposing behavioral AR and IR effects. 
A more convincing interpretation of the effects on P300 amplitude refers again to IOR. It has been suggested above that, unlike empty prime locations, locations activated during presentation of the prime display were subject to IOR at the time of probe display onset. Probe stimuli that appeared in formerly empty locations were more likely to receive attention than were those repeating in formerly occupied locations. Furthermore, it is well established that P300 amplitude elicited by task-relevant stimuli increases with increasing attention. In his influential model of P300 amplitude, Johnson (1986) considered attention to be part of an information transmission factor that is positively correlated with P300 amplitude. Thus, our finding of larger P300 amplitude with control trials than with AR and IR trials is consistent with the notion that IOR is effective in the cognitive processing of both $\mathrm{AR}$ and IR probe displays. With AR trials, the probe target appeared in the prime target location, whereas with IR trials, the probe target appeared in the prime distractor location. Since, during processing of the prime display, more attention is assumed to be devoted to the target than to the distractor, IOR toward the prime target location should be stronger than IOR toward the prime distractor location. As a result, probe targets under the IR condition should receive more attention than do those under the AR condition. This prediction was born out by our data, indicating larger P300 amplitude for IR than for AR trials. It is important to note that this line of reasoning is not at variance with identical P1/N1 amplitude reductions for AR and IR trials as a potential correlate of IOR. Early processing, as reflected by $\mathrm{P} 1$ and $\mathrm{N} 1$ components, presumably did not distinguish between target and distractor stimuli. Thus, P1/N1 reduction most likely reflected the sum of processing costs from both formerly occupied locations, which can be expected to be the same for AR and IR trials. On the other hand, P300 mainly reflected processing of the target stimulus, and therefore, different amplitude reductions for AR and IR trials might well be indicative of differential IOR for locations in which AR and IR probe targets were presented.

\section{LRP Data in the Localization Task}

Finally, LRP data obtained with the localization task may help to clarify why, under the AR condition, marked behavioral PP was observed, although concomitant IOR was assumed to be particularly strong. Despite the fact that the LRP analysis could not be as comprehensive as that with the identification task (see above), a highly similar pattern of LRP waveforms across experimental conditions was revealed for both types of tasks. As with the identification task, atypical LRP involving early positivity and only weak later negativity was found for AR trials, whereas IR and control trials yielded typical LRP waveforms. However, this large difference in the general shape of the LRP is due to the fact that all the AR trials were no-shift trials, whereas most IR and control trials were shift trials. Therefore, rather than the atypically small LRP negativity around the probe response, substantial late LRP negativity, in combination with early LRP positivity, seems to be related to behavioral priming. As was noted earlier, this LRP pattern suggests that on AR trials, after early temporary inhibition, the still activated prime response can simply be repeated, which results in facilitation of RT. Thus, it appeared likely that the mechanism suggested for identity-based PP also applies to location-based PP. When the AR probe target was processed, the considerable advantage of repeating an already activated motor program easily compensated for the slight performance decrement produced by IOR and, moreover, produced a reliable spatial PP effect.

\section{Conclusion}

To sum up, in the present study, motor activation persisting from the preceding prime response was identified as a possible source of PP with both identification and localization tasks. With identification, facilitation of stimulus evaluation may represent an additional process that becomes effective with AR trials. There was no evidence for episodic retrieval of the prime response as a valid explanation of identity-based PP effects. Findings from the localization task were consistent with the notion suggesting that IOR and spatial NP are the same. However, IOR may also be present in spatial PP. In the latter case, IOR counteracts the PP effect produced by central motor activation persisting from the preceding prime response. With regard to location-based priming, our electrophysiological findings argued against both the episodic retrieval and the feature match/mismatch view. A cautionary note, however, is that in the present study, no identity-based NP effect could be established and that no traditional experimental conditions were employed. To reiterate, in the present AR trials, the probe target stimulus/location matched the prime target stimulus/location, and the probe distractor stimulus/location matched the prime distractor stimulus/location. On IR trials, on the other hand, prime target and distractor stimuli/locations were reversed on the probe. These AR and IR conditions were employed because there was evidence that stronger priming effects should result from these modified conditions than from traditional AR and IR conditions, in which only the probe target stimulus/location has a prime history (Stadler \& Hogan, 1996). Furthermore, we assumed that the modified AR and IR conditions would lead to the same conclusions as the traditional ones when predictions derived from the three major priming theories were contrasted. For example, if an episodic retrieval mechanism were responsible for priming effects, essentially the same ERP correlates of priming should have been found regardless of whether there were one or two cues for retrieval of prime information. Nevertheless, it cannot be completely ruled out that, if traditional AR and IR conditions were used, different behavioral and ERP priming effects would have been found.

All in all, the present study has clearly shown that the use of ERP measures represents a promising research strategy for elucidating basic cognitive mechanisms involved in PP and NP. Furthermore, the present data provide further support for the notion that more than a single brain 
process underlies behavioral priming effects. According to our findings, at least two distinct types of processes appear to be involved in location-based priming - that is, attention and motor processes. It is most interesting that these processes may sometimes oppose each other, making it difficult to distinguish them by means of behavioral measures, such as RT.

\section{REFERENCES}

Christie, J., \& KLein, R. M. (2001). Negative priming for spatial location? Canadian Journal of Experimental Psychology, 55, 24-38.

Coles, M. G. H. (1989). Modern mind-brain reading: Psychophysiology, physiology, and cognition. Psychophysiology, 26, 251-269.

ConNelly, S. L., \& Hasher, L. (1993). Aging and the inhibition of spatial location. Journal of Experimental Psychology: Human Perception \& Performance, 19, 1238-1250.

Donchin, E., \& Coles, M. G. H. (1988). Is the P300 component a manifestation of context updating? Behavioral \& Brain Sciences, 11, 357-374

Driver, J., \& TipPeR, S. P. (1989). On the nonselectivity of "selective" seeing: Contrasts between interference and priming in selective attention. Journal of Experimental Psychology: Human Perception \& Performance, 15, 304-314.

Eason, R., Harter, M., \& White, C. (1969). Effects of attention and arousal on visually evoked cortical potentials and reaction time in man. Physiology \& Behavior, 4, 283-289.

EIMER, M. (1994). An ERP study on visual spatial priming with peripheral onsets. Psychophysiology, 31, 154-163.

EIMER, M. (1995). Event-related potential correlates of transient attention shifts to color and location. Biological Psychology, 41, 167-182.

EIMER, M., \& SCHRÖGER, E. (1995). The location of preceding stimuli affects selective processing in a sustained attention situation. Electroencephalography \& Clinical Neurophysiology, 94, 115-128.

ERIKSEN, B. A., \& ERIKSEN, C. W. (1974). Effects of noise letters upon the identification of a target letter in a nonsearch task. Perception \& Psychophysics, 16, 143-149.

Fox, E. (1994). Interference and negative priming from ignored distractors: The role of selection difficulty. Perception \& Psychophysics, 56, $565-574$

Fox, E. (1995a). Negative priming from ignored distractors in visual selection: A review. Psychonomic Bulletin \& Review, 2, 145-173.

Fox, E. (1995b). Pre-cuing target location reduces interference but not negative priming from visual distractors. Quarterly Journal of Experimental Psychology, 48A, 26-40.

Gibbons, H., \& Rammsayer, T. H. (2004). Differential effects of primeprobe duration on positive and negative location priming: Evidence for opponent facilitatory and inhibitory influences in priming tasks. Quarterly Journal of Experimental Psychology, 57A, 61-86.

Gratton, G., Coles, M. G. H., \& Donchin, E. (1983). A new method for off-line removal of ocular artifact. Electroencephalography \& Clinical Neurophysiology, 55, 468-484.

Gratton, G., Coles, M. G. H., Sirevaag, E. J., Eriksen, C. W., \& Donchin, E. (1988). Pre- and poststimulus activation of response channels: A psychophysiological analysis. Journal of Experimental Psychology: Human Perception \& Performance, 14, 331-344.

Henson, R. N. (2003). Neuroimaging studies of priming. Progress in Neurobiology, 70, 53-81.

Hillyard, S. A., LUCK, S. J., \& Mangun, G. R. (1994). The cuing of attention to visual field locations: Analysis with ERP recordings. In H.-J. Heinze, T. F. Münte, \& G. R. Mangun (Eds.), Cognitive electrophysiology (pp. 1-25). Boston: Birkhäuser.

Houghton, G., \& Tipper, S. P. (1994). A model of inhibitory mechanisms in selective attention. In D. Dagenbach \& T. H. Carr (Eds.), Inhibitory processes in attention, memory, and language (pp. 53-112). San Diego: Academic Press.

Huber, D. E., Shiffrin, R. M., Lyle, K. B., \& Ruys, K. I. (2001). Perception and preference in short-term word priming. Psychological Review, 108, 149-182.

JASPER, H. H. (1958). The ten-twenty electrode system of the Interna- tional Federation. Electroencephalography \& Clinical Neurophysiology, 20, 371-375.

Jentzsch, I., \& Sommer, W. (2002). Functional localization and mechanisms of sequential effects in serial reaction time tasks. Perception \& Psychophysics, 64, 1169-1188.

Johnson, R. (1986). A triarchic model of P300 amplitude. Psychophysiology, 23, 367-384.

KIEFER, M. (2002). The N400 is modulated by unconsciously perceived masked words: Further evidence for an automatic spreading activation account of N400 priming effects. Cognitive Brain Research, 13, 27-39.

Logan, G. D. (1988). Toward an instance theory of automatization. Psychological Review, 95, 492-527.

Luck, S. J., Heinze, H. J., Mangun, G. R., \& Hillyard, S. A. (1990). Visual event-related potentials index focused attention within bilateral stimulus arrays: II. Functional dissociation of P1 and N1 components. Electroencephalography \& Clinical Neurophysiology, 75, 528-542.

MacDonald, P. A., \& Joordens, S. (2000). Investigating a memorybased account of negative priming: Support for selection-feature mismatch. Journal of Experimental Psychology: Human Perception \& Performance, 26, 1478-1496.

Mangun, G. R. (1994). Orienting attention in the visual fields: An electrophysiological analysis. In H.-J. Heinze, T. F. Münte, \& G. R. Mangun (Eds.), Cognitive electrophysiology (pp. 81-101). Boston: Birkhäuser.

Mangun, G. R., \& Hillyard, S. A. (1988). Spatial gradients of visual attention: Behavioral and electrophysiological evidence. Electroencephalography \& Clinical Neurophysiology, 70, 417-428.

Mangun, G. R., Hillyard, S. A., \& LuCK, S. J. (1993). Electrocortical substrates of visual selective attention. In D. E. Meyer \& S. Kornblum (Eds.), Attention and performance XIV: Synergies in experimental psychology, artificial intelligence, and cognitive neuroscience (pp. 219-243). Cambridge, MA: MIT Press.

May, C. P., Kane, M. J., \& Hasher, L. (1995). Determinants of negative priming. Psychological Bulletin, 118, 35-54.

McCarthy, G., \& Wood, C. C. (1985). Scalp distributions of eventrelated potentials: An ambiguity associated with analysis of variance models. Electroencephalography \& Clinical Neurophysiology, 62, 203-208.

McDonald, J. J., Ward, L. M., \& KieHL, K. A. (1999). An event-related brain potential study of inhibition of return. Perception \& Psychophysics, 61, 1411-1423.

Miller, J., Patterson, T., \& Ulrich, R. (1998). Jackknife-based method for measuring LRP onset latency differences. Psychophysiology, 35, 99-115.

Milliken, B., Joordens, S., Merikle, P. M., \& Seiffert, A. E. (1998). Selective attention: A reevaluation of the implications of negative priming. Psychological Review, 105, 203-229.

Milliken, B., Tipper, S. P., Houghton, G., \& Lupiáñez, J. (2000). Attending, ignoring, and repetition: On the relation between negative priming and inhibition of return. Perception \& Psychophysics, 62, 1280-1296.

Milliken, B., TipPer, S. P., \& Weaver, B. (1994). Negative priming in a spatial localization task: Feature mismatching and inhibition of distractor location. Journal of Experimental Psychology: Human Perception \& Performance, 20, 624-646.

MorTON, J. (1969). Interaction of information in word recognition. Psychological Review, 76, 165-178.

Morton, J. (1979). Facilitation in word recognition: Experiments causing change in the logogen model. In P. A. Kolers, M. E. Wrolstad, \& H. Bouma (Eds.), Processing visible language (Vol. 1, pp. 259-268). New York: Plenum.

Mulder, G., Wijers, A. A., Brookhuis, K. A., Smid, H. G. O. M., \& Mulder, L. J. M. (1994). Selective visual attention: Selective cuing, cognitive processing, and response processing. In H.-J. Heinze, T. F. Münte, \& G. R. Mangun (Eds.), Cognitive electrophysiology (pp. 2680). Boston: Birkhäuser.

Neill, W. T., \& Mathis, K. M. (1998). Transfer-inappropriate processing: Negative priming and related phenomena. In D. L. Medin (Ed.), Psychology of learning and motivation: Advances in research and theory (Vol. 38, pp. 1-44). San Diego: Academic Press.

NeILL, W. T., \& VALDES, L. A. (1992). The persistence of negative prim- 
ing: Steady-state or decay? Journal of Experimental Psychology: Learning, Memory, \& Cognition, 18, 565-576.

Neill, W. T., Valdes, L. A., Terry, K. M., \& Gorfein, D. S. (1992). Persistence of negative priming: II. Evidence for episodic trace retrieval. Journal of Experimental Psychology: Learning, Memory, \& Cognition, 18, 993-1000.

PARK, J., \& KANWISHER, N. (1994). Negative priming for spatial locations: Identity mismatching, not distractor inhibition. Journal of Experimental Psychology: Human Perception \& Performance, 20, 613-623.

Posner, M. I., \& Cohen, Y. (1984). Components of visual orienting. In H. Bouma \& D. G. Bowhuis (Eds.), Attention and performance X: Control of language processes (pp. 531-556). Hillsdale, NJ: Erlbaum.

Pratt, J., \& Abrams, R. A. (1999). Inhibition of return in discrimination tasks. Journal of Experimental Psychology: Human Perception \& Performance, 25, 229-242.

Ratcliff, R., \& McKoon, G. (1997). A counter model for implicit priming in word identification. Psychological Review, 104, 319-343.

Rossell, S. L., Price, C. J., \& Nobre, A. C. (2003). The anatomy and time course of semantic priming investigated by fMRI and ERPs. Neuropsychologia, 41, 550-564.

RUGG, M. D. (1995). ERP studies of memory. In M. D. Rugg \& M. G. H. Coles (Eds.), Electrophysiology of mind: Event-related brain potentials and cognition (pp. 133-170). London: Oxford University Press.

Schooler, L. J., Shiffrin, R. M., \& RaAijmakers, J. G. W. (2001). A Bayesian model for implicit effects in perceptual identification. Psychological Review, 108, 257-272.

Shapiro, K. L., \& Loughlin, C. (1993). The locus of inhibition in the priming of static objects: Object token versus location. Journal of Experimental Psychology: Human Perception \& Performance, 19, 352-363.

Smid, H. G. O. M., Fiedler, R., \& Heinze, H.-J. (2000). An electrophysiological study of the insertion of overt response choice. Journal of Experimental Psychology: Human Perception \& Performance, 26, 1053-1071.

Stadler, M. A., \& Hogan, M. E. (1996). Varieties of positive and negative priming. Psychonomic Bulletin \& Review, 3, 87-90.

Tenpenny, P. L. (1995). Abstractionist versus episodic theories of repetition priming and word identification. Psychonomic Bulletin \& Review, 2, 339-363

TIPPER, S. P. (1985). The negative priming effect: Inhibitory priming by ignored objects. Quarterly Journal of Experimental Psychology, 37A, 571-590.

Tipper, S. P., Brehaut, J. C., \& Driver, J. (1990). Selection of moving and static objects for the control of spatially directed action. Journal of Experimental Psychology: Human Perception \& Performance, 16, 492-504.
Tipper, S. P., \& Cranston, M. (1985). Selective attention and priming: Inhibitory and facilitatory effects of ignored primes. Quarterly Journal of Experimental Psychology, 37A, 591-611.

VAlle-InClan, F. (1996). The locus of interference in the Simon effect: An ERP study. Biological Psychology, 43, 147-162.

Wood, T. J., \& MiLliken, B. (1998). Negative priming without ignoring. Psychonomic Bulletin \& Review, 5, 470-475.

\section{NOTES}

1. On location-based no-shift IR trials, target and distractor stimuli appeared in the same hemifield, with prime locations reversed on the probe (unilateral probe stimulation- e.g., upper left and bottom left location; see Figure 1). On the other hand, several no-shift control trials involved bilateral probe stimulation. For example, prime stimuli appeared in the bottom two locations (target left), whereas probe stimuli appeared in the upper two locations (target left). Thus, with the localization task, there was a difference with respect to uni- versus bilateral stimulation between IR and control conditions when the trials were subdivided into shift and no-shift trials. This impeded a meaningful comparison of IR and control LRPs separately for shift and no-shift trials, due to differences in ERP asymmetry produced by differential laterality effects.

2. Separate analyses for P1 and N1 amplitudes did not yield significant priming effects. A principal component analysis, however, revealed that P1 and N1 loaded on a common factor. Therefore, the observed priming-related amplitude reductions of both components may be related to the same (set of) brain process(es). From our perspective, this justified a P1-N1 peak-to-peak analysis, which could assess early ERP priming effects more reliably than could single-peak analyses.

3. One might consider temporary activation of the wrong hand as reflecting some kind of alternation bias, which would produce early LRP negativity for shift trials and positivity for no-shift trials. However, the persisting late negativity in the grand-average LRP suggests a repetition bias instead. Still, it might be argued that the repetition bias could be part of an automatic perseverative tendency. An alternation bias, operative shortly after probe onset, could represent a controlled effort to compensate for that perseverative tendency. Although this possibility cannot be completely ruled out on the basis of our data, such an interpretation appears rather unlikely, because late negativity in grand-average LRPs again increased after a temporary decrease around the response. Thus, repetition bias more likely represents a part of controlled activities in preparing for the next display presentation.

(Manuscript received June 26, 2003; revision accepted for publication January 5,2005 .) 